\title{
A Neural Network-Based Model Reference Control Architecture for Oscillation Damping in Interconnected Power System
}

\author{
Waqar Uddin ${ }^{1}{ }^{1}$, Nadia Zeb ${ }^{2}$, Kamran Zeb ${ }^{1,3}{ }^{\circledR}$, Muhammad Ishfaq ${ }^{1}$, Imran Khan ${ }^{1}{ }^{10}$, \\ Saif Ul Islam ${ }^{1}$, Ayesha Tanoli ${ }^{4}$, Aun Haider ${ }^{4}\left(\mathbb{D}\right.$, Hee-Je Kim ${ }^{1, *} \mathbb{1}$ and Gwan-Soo Park ${ }^{1}$ \\ 1 School of Electrical and Computer Engineering, Pusan National University, 2 Busandaehak-ro 63 beon-gil, \\ Geumjeong-gu, Busan-City 46241, Korea; waqudn@pusan.ac.kr (W.U.); kami_zeb@yahoo.com (K.Z.); \\ engrishfaq1994@gmail.com (M.I.); imrankhan@pusan.ac.kr (I.K.); shaheen_575@yahoo.com (S.U.I.); \\ gspark@pusan.ac.kr (G.-S.P.) \\ 2 Department of Electrical Engineering, COMSATS University Islamabad, Abbottabad Campus, Abbottabad \\ 22010, Pakistan; nadia.zeb@gmail.com \\ 3 Department of Electrical Engineering, National University of Science and Technology, Islamabad 44000, \\ Pakistan \\ 4 Department of Electrical Engineering, University of Management and Technology, Lahore, Sialkot Campus, \\ Sialkot 51040, Pakistan; ieesha_tanoli@yahoo.com (A.T.); aun.haider@skt.umt.edu.pk (A.H.) \\ * Correspondence: heeje@pusan.ac.kr; Tel.: +82-10-3492-9677
}

Received: 12 July 2019; Accepted: 20 September 2019; Published: 24 September 2019

\begin{abstract}
In this paper, a model reference controller (MRC) based on a neural network (NN) is proposed for damping oscillations in electric power systems. Variation in reactive load, internal or external perturbation/faults, and asynchronization of the connected machine cause oscillations in power systems. If the oscillation is not damped properly, it will lead to a complete collapse of the power system. An MRC base unified power flow controller (UPFC) is proposed to mitigate the oscillations in 2-area, 4-machine interconnected power systems. The MRC controller is using the NN for training, as well as for plant identification. The proposed NN-based MRC controller is capable of damping power oscillations; hence, the system acquires a stable condition. The response of the proposed MRC is compared with the traditionally used proportional integral (PI) controller to validate its performance. The key performance indicator integral square error (ISE) and integral absolute error (IAE) of both controllers is calculated for single phase, two phase, and three phase faults. MATLAB/Simulink is used to implement and simulate the 2-area, 4-machine power system.
\end{abstract}

Keywords: power oscillations; UPFC; non-linear control; neural network; model reference control

\section{Introduction}

The electric power system is composed of various interconnected generating stations that are connected to load centers through the transmission and distribution systems. The interconnection of different generating stations increases the complexity of the power network. Due to the stochastic nature of the consumer profile, a mismatch occurs between the load demand and supply. The load variation causes a change in system frequency, voltage, active power, and mechanical power. The variation in these parameters causes fluctuation in the rotor speed. As a result, a low frequency $(0.1 \mathrm{~Hz}$ to few hertz) oscillation in active power takes place, known as a power oscillation. These power oscillation either decays after a specified time or increases in magnitude until the system collapse (blackout) occurs [1,2]. 
In order to overcome the issues of stability due to power oscillations and control power flow in a system, a Flexible AC Transmission System (FACTS) has been proposed by scientists and researchers. FACTS devices serve many functions in a power system, i.e., control of power flow, oscillation damping, voltage control, and improvement of transient stability. The Unified Power Flow Controller (UPFC) is an important member of the FACTS family. The UPFC is composed of two voltage source converters coupled through a DC link. The converters provide both series and shunt compensation to the transmission line, hence separating control of active and reactive power becomes possible.

The problem of Low-Frequency Oscillation (LFO) is tackled through FACTS devices in the modern power system. FACT devices and a UPFC based on FACT devices are used mostly for mitigating the LFOs. These devices are also used for enhancing the stability of the power system. The UPFC ensures the security and stability of the system and damps out the LFOs.

Different linear and nonlinear control techniques are applied to mitigate the LFOs [2]. A modified non-dominated sorting genetic algorithm-II (MNSGA-II) is proposed for control of the UPFC to damp out power oscillation in [3]. The proposed design of the UPFC controller is based on a multi-objective optimization problem. However, the controller response is slow in the case of a heavy loaded condition. In [4], the authors presented Variable Neighborhood Search (VNS) optimization algorithm for tackling the problem of oscillations. Although the design process includes high complexity, the proposed controller responds well during normal conditions. However, the author did not consider the heavy loaded or fault condition. An imperialist competition algorithm is used for damping power oscillation in [5]. The controller is designed for wide range of operating conditions, but the author considered a Single Machine Infinite Bus (SMIB) system. The artificial bee colony (ABC) algorithm [6] and Support Vector Regression (SVR) [7] are also proposed for design of a supplementary damping controller. However, the design is equipped with an additional Power System Stabilizer (PSS), along with the UPFC, which will increase system cost and complexity. The author in [8] proposed adaptive input-output feedback linearization control to achieve a non-linear stability estimator and 4th order linearized model. However, due to the parallel application of these procedures, the system faces instability during disturbances. The linear observer excludes the system nonlinearities and only approximate system parameters. The sub-transient state of the synchronous generator is neglected after the fault clearance, while the FACTS device is turned on and off by using an input-output controller. In [9], the researcher proposed a co-evolutionary cultural algorithm (CPCA) based on particle swarm optimization. The author proposed a PSS, along with UPFC, for oscillation damping. But the response of the controller is slow as it takes too much time in damping the power oscillations. In [10], a PSS is used to stabilize the LFOs. Also, in case of failure of the PSS, a supplementary control based on a Linear Quadratic Regulator (LQR) regulates the control input to FACTS devices. In [11], the author proposed a Fuzzy Neural Non-linear Proportional Integral controller for damping LFOs. The positive aspects of this controller are its adaptive nature in learning rate, online tuning, simple structure, and parameter identification. Inter-area oscillations can be efficiently damped through neural predictive control. It will also enhance the stabilization of the power system [12]. A Takagi-Sugeno controller produces gain over the large operating region. However, it cannot guarantee the stability of the system, the robustness of uncertain perturbation in the system, and parameter variations. Transient stability of a machine connected to an infinite bus system is enhanced by using non-linear coordination of generator excitation in [13].

In our paper, we present the neural network (NN)-based model reference controller (MRC) control scheme with a UPFC for mitigation and damping LFOs that is caused by the fault, disturbances, or due to loss of synchronism in connected machines. The MRC controller uses a NN that has the capability to learn and store information of non-linearities, using the auto-regression model instead of tuning of input parameters. The MRC is the integration of the (1) reference model, (2) NN plant, (3) NN controller, and (4) plant. The incorporation of all these four parts promises the robustness, intelligence, and adaptation of parameters. The MRC has an inherently adaptive mechanism, which will help in tracking of variation of process variables. The ability of a NN to learn and model complex 
and nonlinear relationships plays a vital role in control of a complex problem with uncertainties. After initial weight adjustment, it acquires the ability to predict uncertainties. These features of an NN-based MRC controller will tackle the uncertainties and will have a better response than other conventional control schemes. The weight of the NN is adjusted with the Back Propagation (BP) algorithm. Eventually, it will damp out the oscillation quickly and protect the system from complete collapse. Moreover, the performance of proposed controller is compared with the PI controller for the same system to validate our claim.

The paper is organized as follows: Section 2 shows the general system model, while Section 3 discusses the mathematical modelling of the system. The supplementary control and results are discussed in Sections 4 and 5, respectively. Section 6 concludes this paper.

\section{System Model}

A UPFC installed, 2-area, 4-generator connected power system is depicted in Figure 1. This model is used to study LFOs of 0.1 to few Hertz.

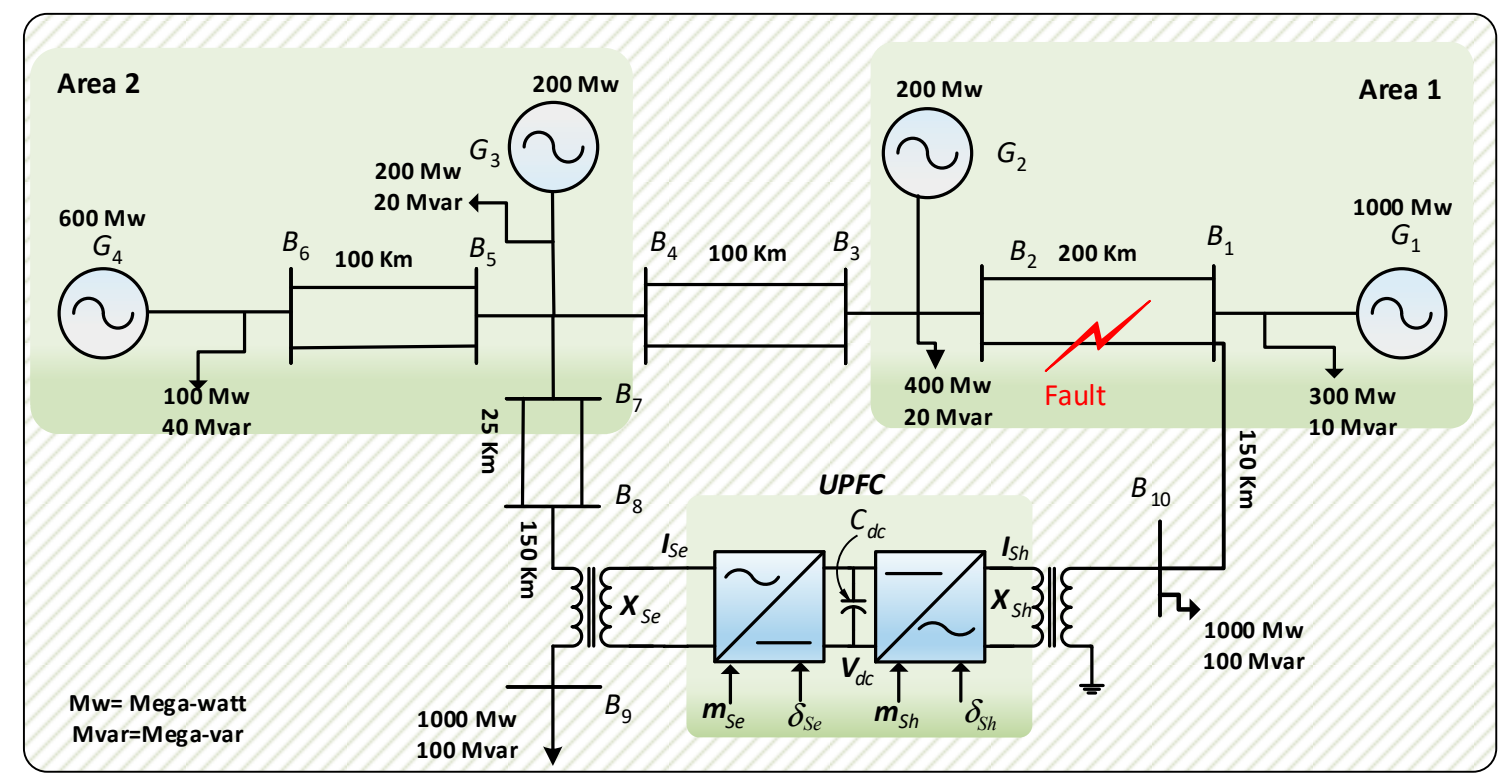

Figure 1. One-line diagram of power system with installed unified power flow controller (UPFC).

A DC capacitor voltage regulator is used to damp out the oscillation produces due to the occurrence of disturbances in power, but it has a negative impact on the generated transients [14] because the required amount of power is transmitted to the series inverter for injection to the transmission line through DC link. Similarly, when variations in magnitude of DC link voltage occurs, it affects power transfer capability of the shunt inverter. Hence, the active power is injected into the system with the help of series inverter [15].

The synchronous generator connected to the system is used to generate power that is delivered through the transmission lines. The installation point of the UPFC is between bus 8 and bus 9 .

The DC link capacitor is used to make the back to back connection of Voltage Sources Converters (VSCs) possible and transfer the power from the shunt converter to the series converter [16]. The main purpose of Series VSC (VSC-SE) is to control the injection of active power and reactive power to the system, while Shunt VSC (VSC-SH) is used to adjust active power flow to series converter and maintain DC link voltage. The connection of the shunt and series VSC is depicted in Figure 1. The connection of both the converters is such that it diminishes the total power injection to the system.

$$
P_{S e}+P_{S h}=0
$$


The input control signal to UPFC are $m_{S e}, m_{S h}, \delta_{S e}$, and $\delta_{S h} . m_{S e}$ and $m_{S h}$ is used to represent the amplitude modulation index of series and shunt compensation, respectively. However, $\delta_{S e}$ and $\delta_{S h}$ are used for the representation of series and shunt compensation phase angles [2,17]. In the case of a series converter, $\delta_{S e}$ and $m_{S e}$ are the important factors that need to be determined. Because $\delta_{S e}$ is used to control real power flow in to the high voltage direct current (HVDC) transmission line, and $m_{S e}$ is responsible for voltage control. Similarly, in the case of the shunt converter, the reactive power of the AC terminal can be controlled through $m_{S h}$, and $\delta_{S h}$ is used to control DC link voltage $\left(V_{d c}\right)$. VSC-SE injects voltage to the AC transmission line through a series connected transformer. This series injected voltage is used to tackle different problems, i.e., phase shift, series compensation, voltage control, and combination of these parameters. VSC-SH is used to take real power from AC terminal and transfer it to DC terminal. This power is then transferred by VSC-SE to the system for compensation. These features make the UPFC a flexible FACTS device that has the properties of all other FACTS devices. The block diagram of the UPFC general control is depicted in Figure 2. $V_{1}$ and $V_{2}$ are bus voltages to which VSC-SH and VSC-SE are connected, respectively, while " $i$ " is line current.

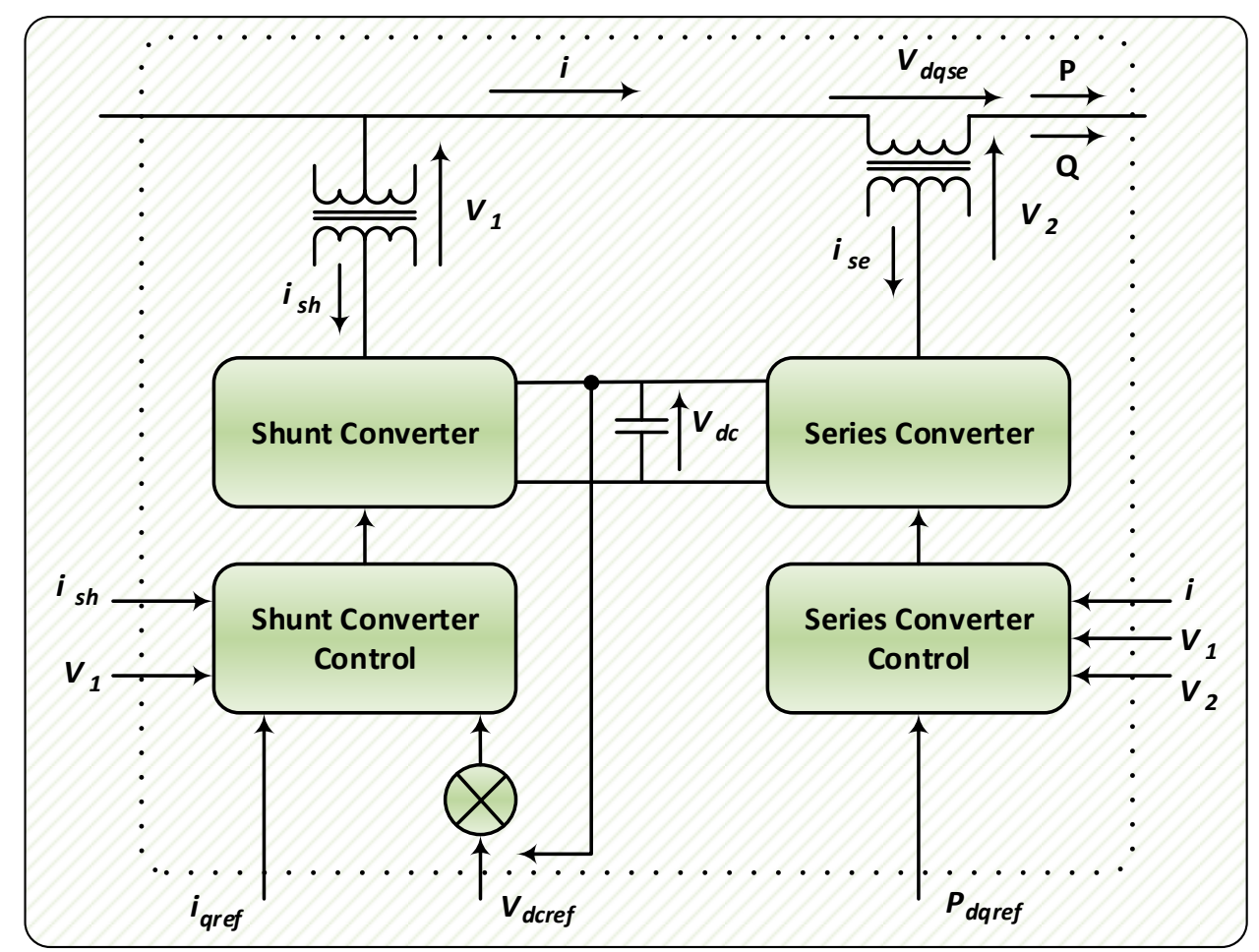

Figure 2. UPFC control.

\section{Mathematical Modelling of Power System}

The model used in this paper is composed of a UPFC installed, 4-machine, two-area power system. The UPFC is composed of a VSC - SH, VSC - SE, and DC link.

\section{Mathematical Model}

The non-linear model of the UPFC is presented below from Equation (2) to Equation (3). This model is used for the analysis of stability of the power system. The parameters that affect the dynamics of the system are considered in this model, and resistance and transients of transformer is neglected. The modelling equations given below are transformed to dq - axis by using Park's transformation $[17,18]$. Series VSC and shunt VSC are modelled as:

$$
\left[\begin{array}{c}
V_{d s e} \\
V_{q s e}
\end{array}\right]=\left[\begin{array}{cc}
0 & -X_{s e} \\
X_{s e} & 0
\end{array}\right]\left[\begin{array}{c}
I_{d s e} \\
I_{q s e}
\end{array}\right]+\frac{m_{s e} V_{d c}}{2} *\left[\begin{array}{c}
\cos \delta_{s e} \\
\sin \delta_{s e}
\end{array}\right]
$$




$$
\left[\begin{array}{c}
V_{d s h} \\
V_{q s h}
\end{array}\right]=\left[\begin{array}{cc}
0 & -X_{s h} \\
X_{s h} & 0
\end{array}\right]\left[\begin{array}{c}
I_{d s h} \\
I_{q s h}
\end{array}\right]+\frac{m_{s h} V_{d c}}{2} *\left[\begin{array}{c}
\cos \delta_{s h} \\
\sin \delta_{s h}
\end{array}\right]
$$

The subscript 'sh' and 'se' is used for shunt and series converter parameters, respectively. $X_{s e}, X_{s h}$ are equivalent reactance of series and shunt transformer. $V_{d s e}, V_{q s e}$ are the dq voltage component of series branch, while $I_{d s e}, I_{q s e}$ are $d q$-component of series converter current. The dynamic of DC voltage at DC link is represented as:

$$
\frac{d v_{d c}}{d t}=\frac{3 m_{s h}}{4 C_{d c}}\left[\begin{array}{cc}
\cos \delta_{s h} & \sin \delta_{s h}
\end{array}\right]\left[\begin{array}{c}
I_{d s h} \\
I_{q s h}
\end{array}\right]+\frac{3 m_{s e}}{4 C_{d c}}\left[\begin{array}{cc}
\cos \delta_{s e} & \sin \delta_{s e}
\end{array}\right]\left[\begin{array}{c}
I_{d s e} \\
I_{q s e}
\end{array}\right] .
$$

$V_{d c}$ shows the DC link voltage, and $C_{d c}$ is the capacitances of the DC link capacitor. The model of the UPFC connected power system is presented by:

$$
\begin{gathered}
\dot{\delta}=\omega_{0} \Delta \omega, \\
\dot{\omega}=\frac{P_{\text {mech }}-P_{\text {elec }}-D \Delta \omega}{M}, \\
\dot{E_{f d}}=-\frac{E_{f-d}}{T_{A}}+\frac{K_{A}}{T_{A}}\left(V_{s 0}-V_{s}\right), \\
\dot{E_{s q}^{\prime}}=\frac{E_{f-d}-\left(x_{d}-x_{d}^{\prime}\right) i_{d}-E_{s q}^{\prime}}{T_{d 0}^{\prime}} .
\end{gathered}
$$

$P_{\text {mech }}$ and $P_{\text {elec }}$ represent mechanical and electrical power, respectively, where $M$ and $D$ represent inertia and damping coefficient, respectively. $\omega_{0}$ represents synchronous speed. $\delta$ and $\omega$ are rotor angle and rotor speed, respectively, while $\Delta \omega=(\omega-1) . E_{f d}, E_{s q}^{\prime}$, and $V$ are field, internal, and the terminal voltage of generator. $T_{d 0}^{\prime}$ represents the time constant of the open circuit. $T_{A}$ and $K_{A}$ are time constant and exciter gain, respectively, while $V_{s 0}$ represents reference voltage. $x_{d}$ is the $d$-axis reactance of generator, and $x_{d}^{\prime}$ is transient reactance. Furthermore, the different terms used in Equation (5) to Equation (8) are given as:

$$
\left.\begin{array}{c}
P_{\text {elec }}=V_{s-q} I_{s-q}+V_{s-d} I_{s-d} \\
V_{s}=\sqrt{V_{d}^{2}+V_{q}^{2}} \\
V_{d}=X_{d} I_{q}, V_{q}=E_{q}^{\prime}-X_{d}^{\prime} I_{d} \\
I_{d}=I_{E d}+I_{B d}, I_{q}=I_{E q}+I_{B q}
\end{array}\right\} .
$$

Similarly, the line current for VSC-SE and VSC-SH can be obtained as [17-19]:

$$
\begin{gathered}
I_{s e d}=-\frac{X_{s h}}{X_{d} \Sigma} E_{q}^{\prime}+\frac{X_{s h d} m_{s h} \sin \delta_{s h} V_{d c}}{2 X_{d} \Sigma}-\frac{X_{t d}}{X_{d} \Sigma}\left(\frac{m_{s e} \sin \delta_{s e} V_{d c}}{2}+V_{b} \cos \delta\right), \\
I_{s e q}=-\frac{X_{s h q} m_{s h} \sin \delta_{s h} V_{d c}}{2 X_{q} \Sigma}+\frac{X_{s h q}}{X_{q} \Sigma}\left(\frac{m_{s e} \sin \delta_{s e} V_{d c}}{2}+V_{b} \sin \delta\right), \\
I_{s h d}=\frac{X_{s e}}{X_{d} \Sigma} E_{q}^{\prime}-\frac{X_{s e d} m_{s h} \sin \delta_{s h} V_{d c}}{2 X_{d} \Sigma}+\frac{X_{s h d}}{X_{d} \Sigma}\left(\frac{m_{s h} \sin \delta_{s h} V_{d c}}{2}+V_{b} \cos \delta\right), \\
I_{s h q}=-\frac{X_{s h q} m_{s h} \cos \delta_{s h} V_{d c}}{2 X_{q} \Sigma}+\frac{X_{t q}}{X_{q} \Sigma}\left(\frac{m_{s e} \cos \delta_{s e} V_{d c}}{2}+V_{b} \sin \delta\right),
\end{gathered}
$$

where as $X_{d} \Sigma, X_{t d}, X_{q} \Sigma$, and $X_{t q}$ are defined in [18]. 


\section{Supplementary Control Design}

Supplementary control schemes with the MRC are introduced for mitigating LFOs in the power system. The control design is used in the UPFC internal control structure to enhance the stability of the power system. As the faults occur on the transmission system, the speed of a different generator connected to the power system deviates and become out of synchronism. This speed deviation $(\Delta \omega)$ is treated as an input to the supplementary controller, as depicted in Figure 3. The controllers mitigate the LFOs by providing suitable input to the UPFC, hence improving the power quality and stabilizing the power system. The design of the controllers is based on model reference neural control, and it uses a nonlinear autoregressive moving average model. The MRC is the integration of the (1) reference model, (2) NN plant, (3) NN controller, and (4) plant. The different parts of controller will be discussed in detail in a subsection of this section.

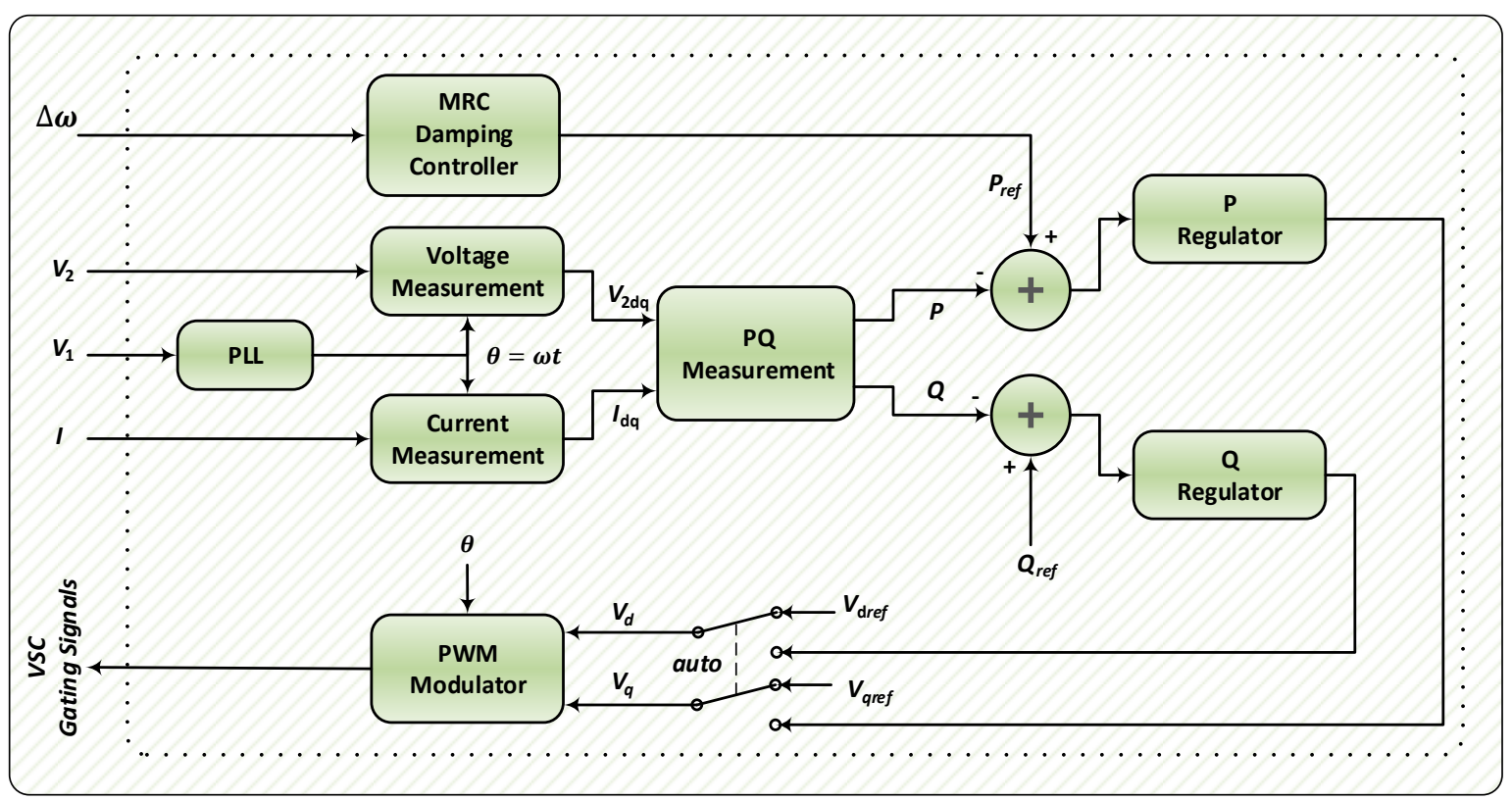

Figure 3. Control structure for oscillation damping. $\mathrm{MRC}=$ model reference controller; VSC $=$ Voltage Sources Converters; PQ = Active Power, Reactive Power; PWM = Pulse Width Modulation; PLL = Phase Lock Loop.

\subsection{Neural Network (NN)}

The NN, inspired by the biological system, is composed of interconnected building blocks known as neurons. The interconnection weight quantifies the strength of interconnection. Because of these interconnected weights, the $\mathrm{NN}$ acquires the ability to learn and model complex relationships. Hence, it becomes more intelligent in predicting the uncertainties. Due to the learning capability of NN, it has a wide range of applications in automation and optimization [20], image processing [21,22], speech recognition [23,24], control [25,26], modelling, and time series prediction [27,28].

The architecture of $\mathrm{NN}$ is composed of multiple input layer and hidden layers, as depicted in Figure 4. This architecture is known as " $N-1$ " feedforward NNs. Whereas ' $N$ ' represents the number of hidden neurons and it has only one output node. The design of the architecture of NN depends on the input weights ' $z_{i j}$ ' and output weights ' $\gamma_{i}$ '. The formulation of the $\mathrm{NN}$ through the nonlinear function of hidden layer activation function ' $\psi_{i}$ ' is presented as:

$$
\hat{y}(k+1)=\sum_{i=1}^{N} \gamma_{i} \psi_{i}\left(\sum_{j=1}^{n+m} z_{i j} X_{j}\right),
$$


where $X=\left[u_{1}, u_{2}, \ldots, u_{m+n}\right]$ is the input vector to $\mathrm{NN}$ and ' $\psi_{i}$ ' is the activation function of the ' $i$ 'th' hidden layer. The neuron in the hidden layer uses the nonlinear logistic function given by Equation (15):

$$
\psi_{i}\left(\sum_{j=1}^{n+m} z_{i j} X_{j}\right)=\frac{1}{1+\exp \left(\sum_{j=1}^{n+m} z_{i j} X_{j}\right)} .
$$

The equation can be rearranged as:

$$
\hat{y}(k+1)=\theta^{T} \psi
$$

whereas $\psi \triangleq\left(\psi_{1}, \psi_{2}, \ldots, \psi_{N}\right)^{T}, \theta^{T} \triangleq\left(\gamma_{1}, \gamma_{2}, \ldots, \gamma_{N}\right)$. The gradient descent optimization technique is used to tune and update the output weight only. This will, in turn, reduce the computational complexity of the NN. The update equation of the NN is given as:

$$
\gamma_{k+1}=\gamma_{k} \alpha\left(\left(y_{r} \times \hat{y}\right) \times\left(y_{r} \times \hat{y}\right)^{\prime}\right) .
$$

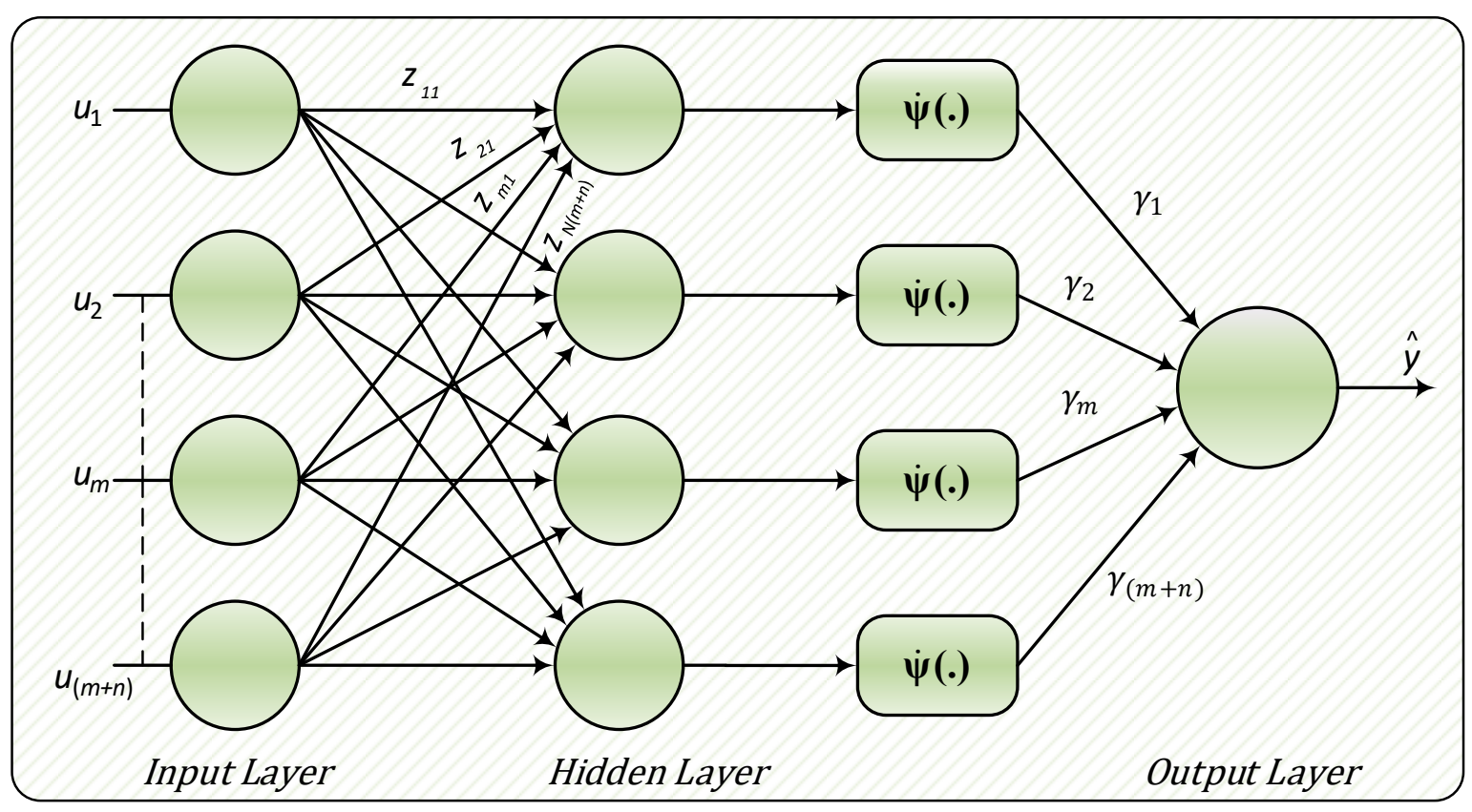

Figure 4. Neural network (NN) architecture.

\subsection{Model Reference Controller}

The NN controller is used in the MRC for tracking the reference signal, as well as for plant identification. The MRC uses two NN controllers and minimal online computation. One NN is used to train the controller, and the other is used for plant identification. The data set for training the NN controller is given in Appendix A. The architecture of the MRC is depicted in Figure 5. The controller produces a controlled output signal $u(t)$ that acts as input to the plant. This control signal brings the output signal $y_{p}(t)$ closer to the reference signal $y_{r}(t)$.

According to [29], the different inputs and their respective outputs define the characteristics of the real-time process. The previous information of input and output is used to introduce system dynamics. Equation (18) is sampled at a regular interval of time:

$$
y_{p}(t)=f_{p}\left[y_{p}(t-1), \ldots y_{p}\left(t-n_{a}\right), u(t-k), \ldots u\left(t-k-n_{b}\right)\right]
$$


where $k \geq 1$ shows the time delay in number of samples, $f_{p}[(y, u)]$ is processed by non-linearity, and $n_{a}$, $n_{b}+1$ represents the process output and input, respectively. Equation (18) gets into the shape of the transfer function below by linearizing the process about the operating point.

$$
\frac{y_{p}(t)}{u(t)}=q^{-k} \frac{B\left(q^{-1}\right)}{A\left(q^{-1}\right)}=q^{-k} \frac{b_{0}+b_{1} q^{-1}+\ldots+b_{n_{b}} q^{-n_{b}}}{1+a_{1} q^{-1}+\ldots+a_{n_{a}} q^{-n_{a}}} .
$$

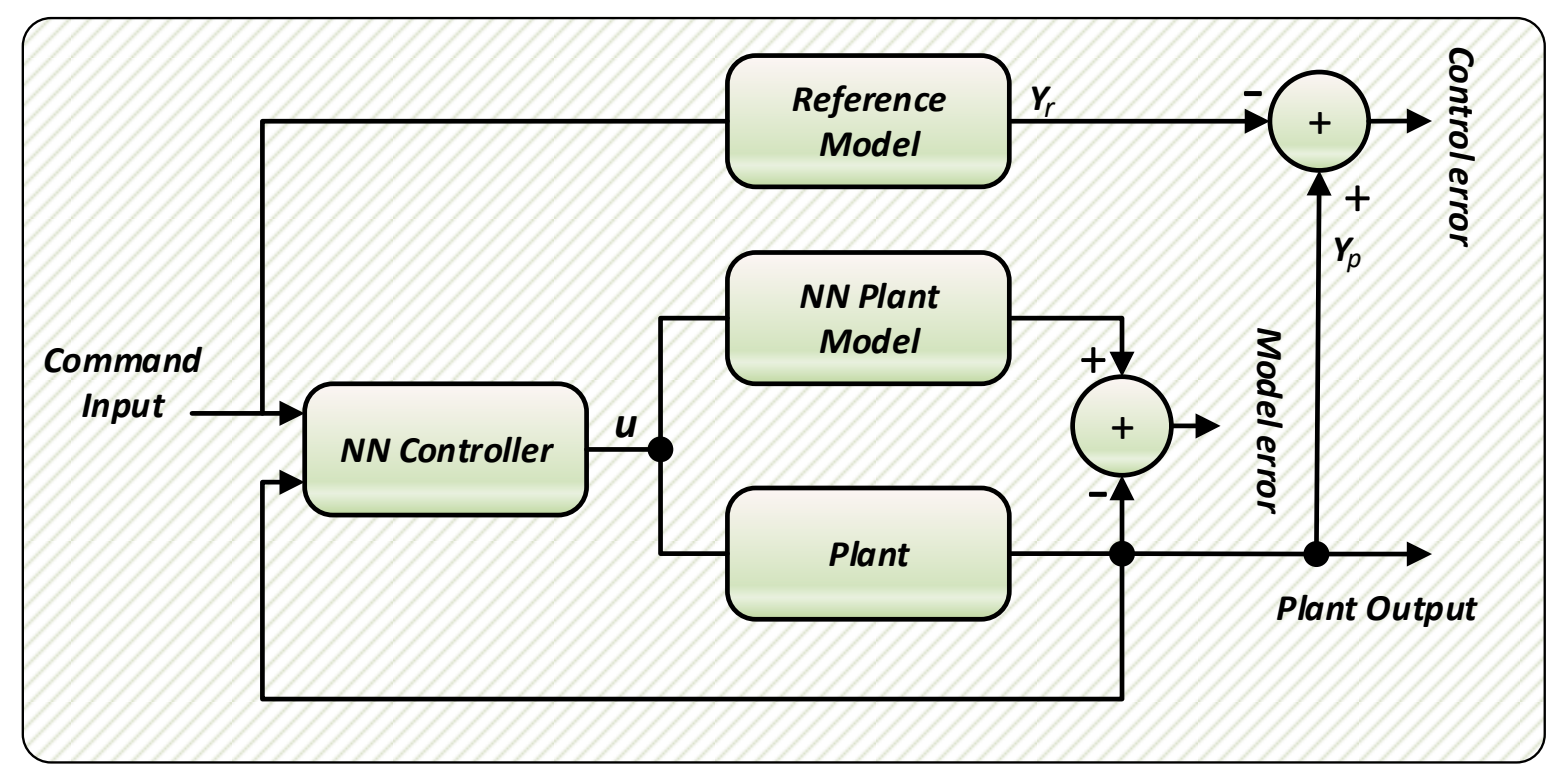

Figure 5. NN-based MRC.

In Equation (19), $y_{p}(t)$ and $u(t)$ represents the deviation of process from its operating points. The parameters $a_{i}$ and $b_{i}$ are dependent on the operating condition, while $q^{-1}$ represents the backward shift operator. The equation for the generalized linear controller is given in Equation (20)

$$
u(t)=\frac{1}{F\left(q^{-1}\right)}\left(H\left(q^{-1}\right) R(t)-G\left(q^{-1}\right) y_{p}(t)\right),
$$

where ' $R$ ' is set point, while $F, G$, and $H$ are the polynomial defined below:

$$
\left\{\begin{array}{c}
F\left(q^{-1}\right)=1+f_{1} q^{-1}+\ldots+f_{n_{f}} q^{-n_{f}} \\
G\left(q^{-1}\right)=g_{0}+g_{1} q^{-1}+\ldots+g_{n_{g}} q^{-n_{g}} \\
H\left(q^{-1}\right)=h_{0}+h_{1} q^{-1}+\ldots+h_{n h} q^{-n h}
\end{array}\right.
$$

The closed-loop transfer function of a linearized system is given by:

$$
\frac{y_{p}(t)}{R(t)}=q^{-k} \frac{B\left(q^{-1}\right) H\left(q^{-1}\right)}{A\left(q^{-1}\right) F\left(q^{-1}\right)+q^{-k} B\left(q^{-1}\right) G\left(q^{-1}\right)} .
$$

Equation (20) and the control structure $n_{f}, n_{g}$ and $n_{h}$ of Equation (21) is used to design a linear controller model to track the reference point. The polynomial B is neglected to make Equation (22) equal to the characteristic equation of the system. Hence, $B$ is replaced with another term, i.e., $B\left(q^{-1}\right)=$ $b_{0} B^{+}\left(q^{-1}\right)$. Equation (22) will transform into the following equation:

$$
\frac{y_{p}(t)}{R(t)}=q^{-k} \frac{b_{0} H\left(q^{-1}\right)}{A\left(q^{-1}\right) F\left(q^{-1}\right)+q^{-1} b_{0} G\left(q^{-1}\right)}
$$




$$
\frac{y_{p}(t)}{R(t)}=q^{-k_{m}} \frac{s_{0}+s_{1} q^{-1}+\ldots+s_{n_{s}} q^{-n_{s}}}{1+t_{1} q^{-1}+\ldots+t_{n_{t}} q^{-n t}}
$$

$S\left(q^{-1}\right)$ and $T\left(q^{-1}\right)$ are the polynomial that is used to set closed-loop response to a desired value. $k_{m}$ is the time delay of the reference model. In order to enable the cancellation of $B^{+}\left(q^{-1}\right), F\left(q^{-1}\right)$ is replaced with $B^{+}\left(q^{-1}\right) F_{1}\left(q^{-1}\right)$, i.e., $F\left(q^{-1}\right)=B^{+}\left(q^{-1}\right) F_{1}\left(q^{-1}\right)$. The minimal order of the unique controller solution is:

$$
n_{g}=n_{a}-1, n_{f}=n_{b}+k-1, k_{m}=k, \text { while } H\left(q^{-1}\right)=\frac{1}{b_{0}} S\left(q^{-1}\right)
$$

and

$$
n_{t} \leq n_{a}+k-1 .
$$

Hence, the structure of process $n_{a}$ and $k$ is used to limit the order of denominator of closed-looped transfer function $n_{t}$. The order of $n_{t}$ should fulfill the condition given in Equation (25). The lower order of denominator is possible by making unused $t_{i}$ coefficient equal to zero. The following Equation (26) is used to introduce an observer into the controller design.

$$
C\left(q^{-1}\right)=1+c_{1} q^{-1}+c_{2} q^{-2}+c_{n_{c}} q^{-n_{c}}
$$

while

$$
n_{c} \leq n_{a}+k-1-n_{t}
$$

Then, Equation (25) is updated as:

$$
H\left(q^{-1}\right)=\frac{1}{b_{0}} V\left(q^{-1}\right) C\left(q^{-1}\right) .
$$

Although due to nonlinearity, the parameter of controller will change, but the controller designed structure in Equation (20) will not change for any operating point of the plant in Equation (18) despite setting the observer polynomial $C\left(q^{-1}\right)=1$ and $t_{i}$ coefficient to zero. As in Equation (20), a nonlinear controller design with the same input and output can smoothly interpolate between different parameters of linear controllers as the operating points of the plant changes. The MRC in Equation (29) is proposed by considering Equation (20) in difference equation form and linearizing a nonlinear system into the form of Equation (20)

$$
u(t)=f_{r}\left[u(t-1), \ldots u\left(t-n_{f}\right), r_{f}(t), y_{p}(t), \ldots y_{p}\left(t-n_{g}\right)\right] .
$$

In Equation (29), the NN will be used to realize $f_{r}[\mathbf{\bullet}]$, and Equation (24) will define the order of $n_{g}$ and $n_{f}$, while Equation (27) defines $n_{c}$ and $r_{f}(t)=S\left(q^{-1}\right) C\left(q^{-1}\right) r(t)$.

\section{Controller Adaption with BP}

The gradient descent algorithm with time delay is implemented to update the parameter of NN. The output of NN with a multilayer of a neuron is given as:

$$
u(t)=f_{0}\left(\sum_{j} z_{j}^{2}\left(f_{h}\left(\sum_{j} z_{i j}^{1} x_{i}(t)+b_{j}^{1}\right)\right)+b^{2}\right) .
$$

$x_{i}$ is the input to the $\mathrm{NN}$, and $b$ and $z$ are biases and weights of the $\mathrm{NN}$, respectively. $f_{0}$ represents the nonlinear function of the input layer, and $f_{h}$ is function of the hidden layer. The cost function that needs to be minimized is given as:

$$
J(t)=\frac{1}{2} e(t j)^{2}=\frac{1}{2}\left(y_{r}(t)-y_{p}(t)\right)^{2}
$$


$y_{r}$ and $y_{p}$ are the reference and measured output of the system, respectively. The weight parameter ' $z$ ' is used in opposite direction in cost function gradient to minimize the error, given as:

$$
z(t)=z(t-1)-\eta \frac{\partial J(t)}{\partial z} .
$$

The unknown ' $\eta$ ' represents the learning rate. The BP algorithm is used to calculate the gradient algorithm of Equation (32) Hence, Equation (18) and $\frac{\partial J(t)}{\partial y_{p}(t)}=-e(t)$ gives:

$$
\frac{\partial J(t)}{\partial z}=-e(t) \frac{\partial y_{p}(t)}{\partial u(t-k)} \frac{\partial u(t-k)}{\partial z} .
$$

In Equation (33), the first derivative is called process Jacobian. The second NN is used to estimate it as a process model, i.e., the NN model given by:

$$
y^{m}(t)=f_{m}[\boldsymbol{\bullet}]=f_{0}\left\{\sum_{j} z_{j}^{2 m}\left(f_{h}\left(\sum_{i} z_{i j}^{1 m} x_{i}^{m}(t)+b_{j}^{1 m}\right)\right)+b^{2 m}\right\} .
$$

The delayed model inputs/outputs are represented by $x_{i}^{m}(t)$, as shown in Equation (18) in the form of $u(t-k)$. Equation (35) shows one of the estimations of Jacobian.

$$
\frac{\partial y_{p}(t)}{\partial u(t-k)} \approx \frac{\partial y^{m}(t)}{\partial u(t-k)}=f_{0}^{\prime} \sum_{j} z_{j}^{2 m} f_{h_{j}}^{\prime} z_{1 j}^{1 m} .
$$

The process sign is used to approximate Jacobian if the model is not available. Equation (36) shows the update rule for the different parameter of the controller.

$$
z(t)=z(t-1)+\eta e(t) \operatorname{sgn}\left(\frac{\partial y_{p}(t)}{\partial u(t-k)}\right) \frac{\partial u(t-k)}{\partial z} .
$$

This equation is used to update the rule to get better dynamics of the system.

\section{Results and Discussion}

The performance of the designed controller is evaluated and compared with the PI controller under symmetrical and asymmetrical faults. The faults are applied for $50 \mathrm{~ms}$, i.e., the fault is initiated is at $t=200 \mathrm{~ms}$ and cleared at $t=250 \mathrm{~ms}$. Moreover, the fault is applied on the mid-point of the lower line of the double circuit line between buses $B_{1}$ and $B_{2}$, as depicted in Figure 1 . The proposed controller has the capability to mitigate the LFOs and enhance the stability of the power system. The parameters used for comparison of the proposed control structure are: (1) load bus, voltage, (2) speed deviation $(\Delta w)$, and (3) performance indices of controllers.

\subsection{Asymmetrical Faults}

Both asymmetrical and symmetrical faults are applied to the system for $50 \mathrm{~ms}$ (at $t=200 \mathrm{~ms}$ ) to create oscillations in the system. The fault creates a voltage dip of $10 \%$ during the $1-\Phi$ fault, while a $25 \%$ dip is created during the 2- $\Phi$ to ground fault. After the clearance of the 1- $\Phi$ fault, the voltage profile is restored at $t=2 \mathrm{~s}$ due to the adaptive and predictive nature of MRC, while the response with conventional PI is oscillatory and oscillation is damped out after $t=12 \mathrm{~s}$, as shown in Figures 6 and 7, respectively. Similarly, the speed deviation shows high amplitude oscillation in the case of the PI controller, while the MRC has the capability to damp out the oscillation in comparatively less time. Figure 8 shows that the MRC has damped out the oscillation at $t=2 \mathrm{~s}$, and speed deviation $(\Delta w \approx 0)$ is approximately zero, while in comparison with MRC, the PI controller deviates from zero till $t=6 \mathrm{~s}$, as depicted in Figure 8. 


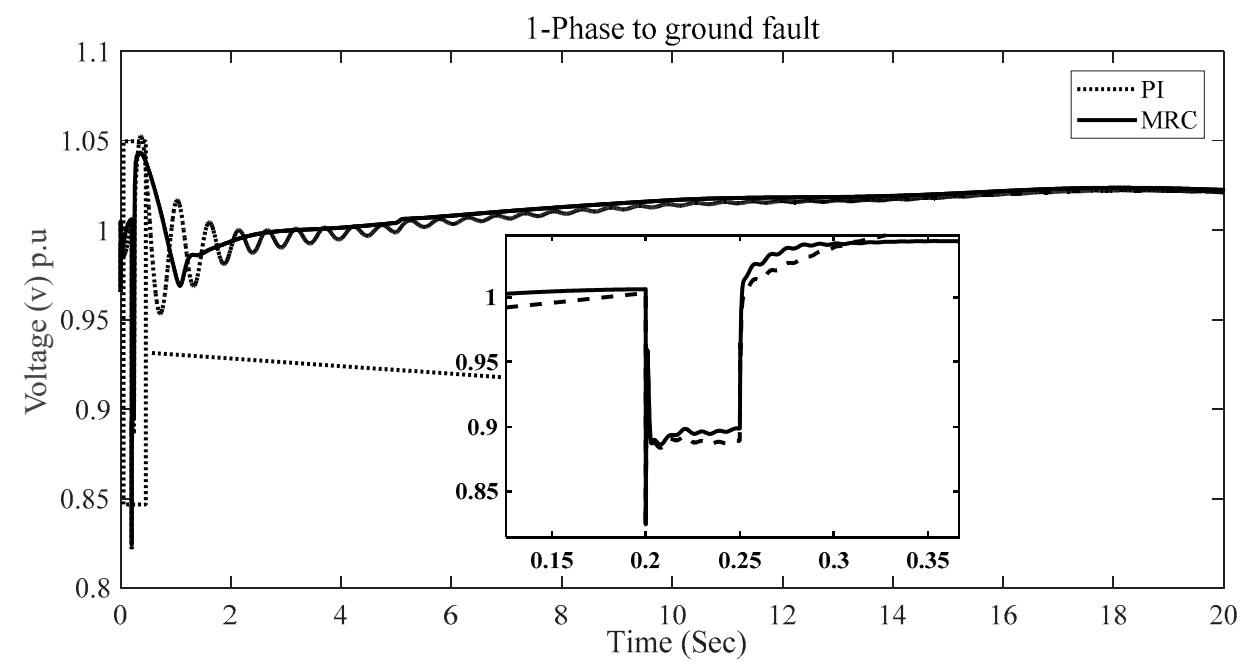

Figure 6. Load bus voltage 1- $\Phi$ to ground fault.

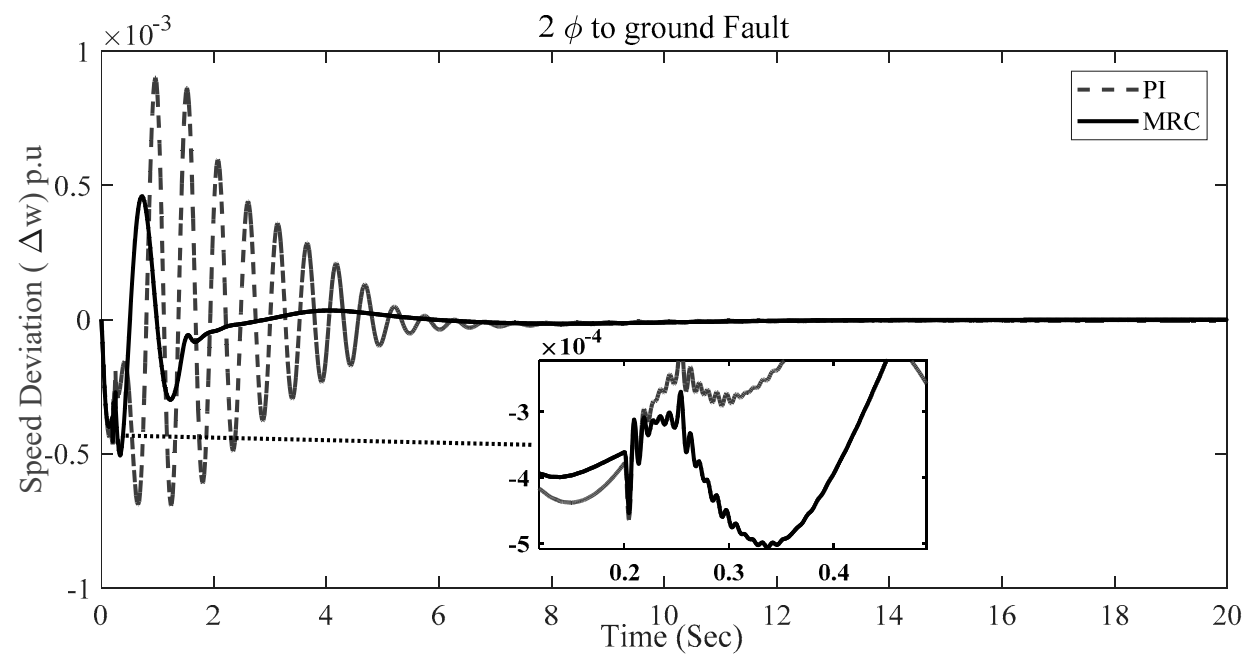

Figure 7. Speed deviation during 2- $\Phi$ to ground fault.

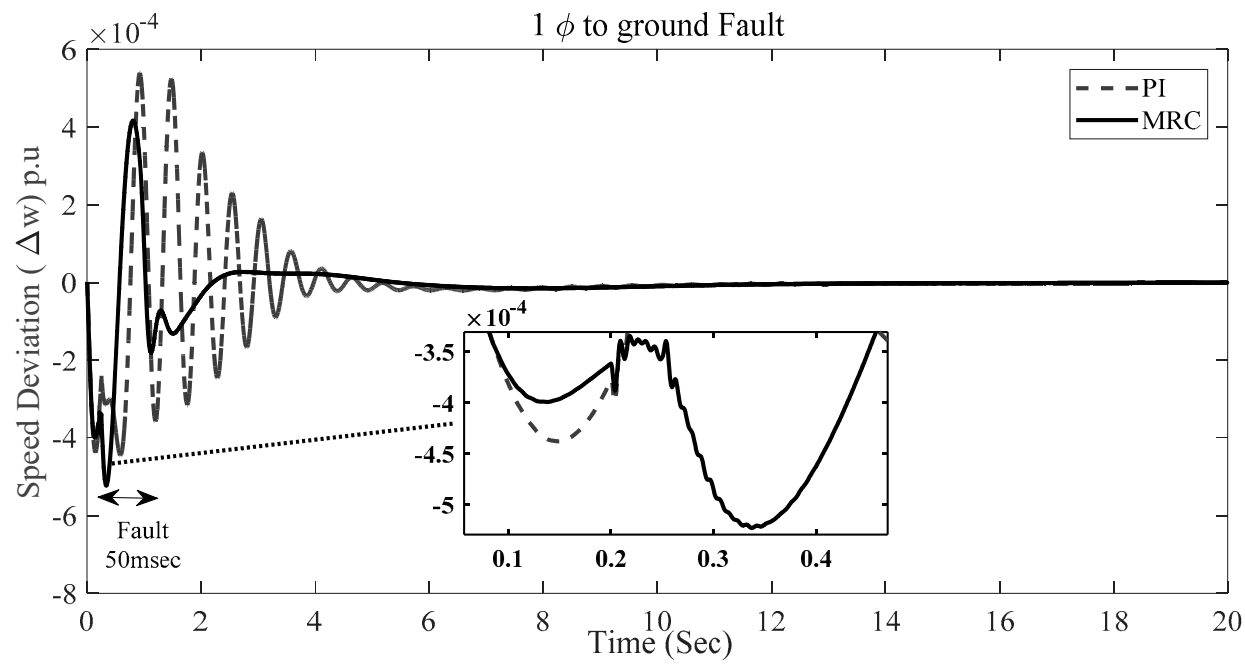

Figure 8. Speed deviation during 1- $\Phi$ to ground fault.

Similarly, during the 2- $\Phi$ to ground fault, the MRC has a better response in mitigation of oscillation as compared to the PI. As depicted in Figure 9, the MRC has smoothed the voltage response abruptly 
after fault clearance at $t=2.5 \mathrm{~s}$, while the PI controller failed to show the robustness. The oscillation is completely damped out at $t=12 \mathrm{~s}$, as shown in Figure 7. Similarly, speed deviation becomes zero at $t=2 \mathrm{~s}$ in the case of the MRC, while the PI vanishes the oscillations at $t=8 \mathrm{~s}$.

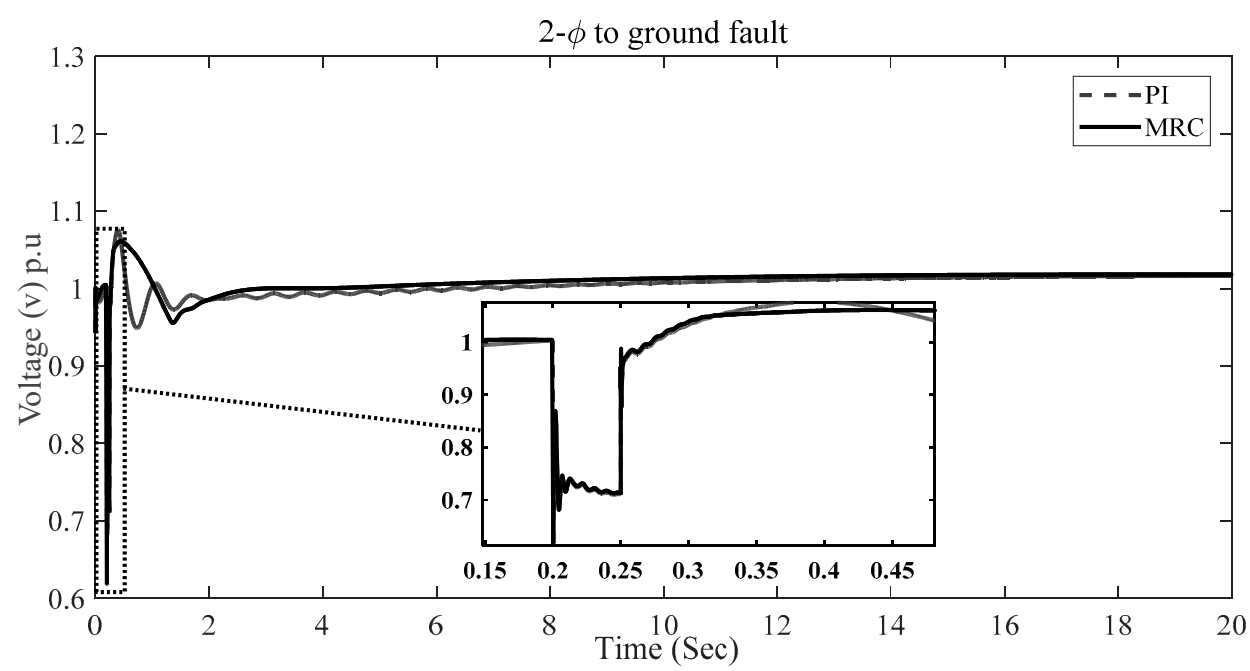

Figure 9. Load bus voltage 2- $\Phi$ to ground fault.

\subsection{Symmetrical Faults}

In the case of an asymmetrical fault, the 3- $\Phi$ to ground fault is applied to evaluate the performance of the proposed controller, as depicted in Figure 10. The MRC has a better approach toward the mitigation of oscillations occurring after the voltage dip, as shown in Figure 11. The oscillation is completely damped out at $t=2.5 \mathrm{~s}$ in the case of the MRC, while the PI has low amplitude oscillations till $t=8 \mathrm{~s}$. Similarly, speed deviation is subjected to larger amplitude high frequency oscillation and higher settling time using the PI, while the proposed controller settles the response to zero at $t=3 \mathrm{~s}$.

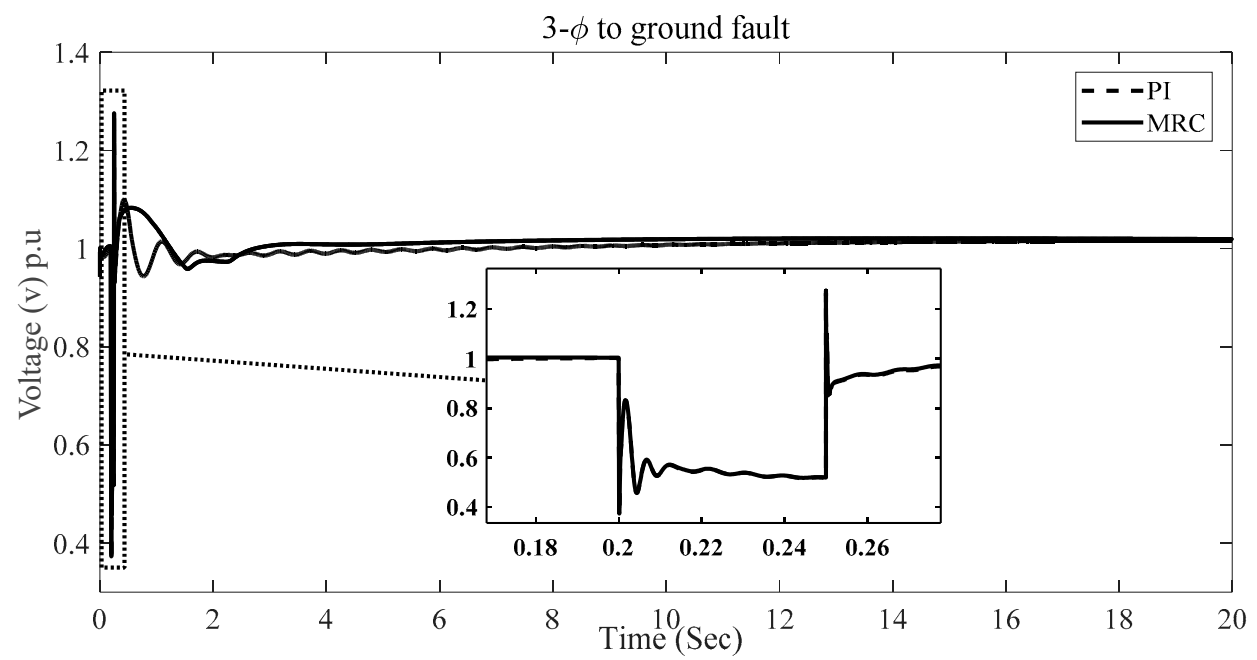

Figure 10. Load bus voltage 3- $\Phi$ to ground fault. 


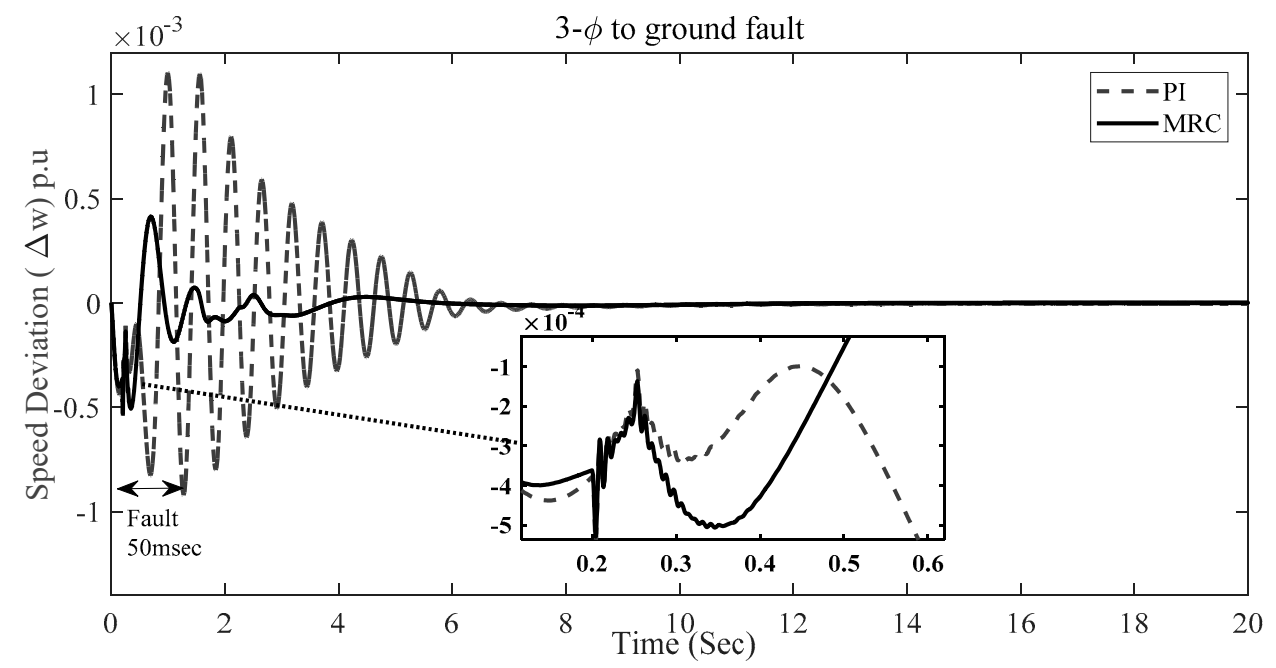

Figure 11. Speed deviation during 3- $\Phi$ to ground fault.

Besides other performance evaluation parameter, Table 1 shows the performance indices of both controllers during all three types of faults. The lower value of integral square error (ISE) and integral absolute error (IAE) shows better response of controller. The MRC controller has both a lower value of ISE error and IAE.

Table 1. Performance indices. ISE = integral square error; IAE = integral absolute error.

\begin{tabular}{ccccccc}
\hline \multirow{2}{*}{ Controllers } & \multicolumn{2}{c}{ 1- $\boldsymbol{\Phi}$ Fault } & \multicolumn{2}{c}{ 2- $\boldsymbol{\Phi}$ Faults } & \multicolumn{2}{c}{ 3- $\boldsymbol{\Phi}$ Fault } \\
\cline { 2 - 7 } & ISE & IAE & ISE & IAE & ISE & IAE \\
\hline PI & 0.0009028 & $2.432 \times 10^{-7}$ & 0.001574 & $6.569 \times 10^{-7}$ & 0.00253 & $1.07 \times 10^{-6}$ \\
MRC & 0.0005561 & $1.301 \times 10^{-7}$ & 0.0005802 & $1.41 \times 10^{-7}$ & 0.0005248 & $1.111 \times 10^{-7}$ \\
\hline
\end{tabular}

\section{Conclusions}

The mitigation of power oscillations is a demanding task to enhance the stability of the power system. An intelligent, optimized, and robust design of the UPFC plays a vital role in damping of these oscillations. With these abilities, the MRC controller has shown tremendous performance in mitigating power oscillations, enhancing system stability, and improving the capability of the control system. Moreover, our control scheme shows robustness and intelligence during system perturbation. In order to show the effectiveness of proposed control scheme, it was compared with a traditional PI controller. The results clearly show the superiority of the NN-based MRC over the traditional PI controller, as it is better in oscillation damping. The NN-based MRC diminishes the amplitude of power oscillation, produced due to fault.

However, the MRC-based on a NN is complex algorithm. Due to its complexity, it requires considerable amount of time for calculation and implementation. Due to the integration of the NN controller in the MRC frame, it is associated with several issues and complications. The complications include selecting the input and output of the controller, as well as training of the $\mathrm{NN}$ for its weight adjustment.

Moreover, soon the proposed control will be extended to more than a 2-area system with a greater number of generation stations. Additionally, a new control scheme will be designed, and its results will be compared with both the PI and the MRC.

Author Contributions: W.U., N.Z., and K.Z. propose the main idea of the paper. W.U., M.I., S.U.I., A.T. and A.H have implemented the mathematical derivations, simulation verifications, and analyses. The paper is written by W.U. and is revised by N.Z., I.K., A.H., and H.-J.K. All the authors were involved in preparing the final version of this manuscript. Besides, this whole work is supervised and funded by H.-J.K. and G.-S.P. 
Funding: This research was supported by Basic Research Laboratory through the National Research Foundations of Korea funded by the Ministry of Science, ICT and Future Planning (NRF-2015R1A4A1041584).

Conflicts of Interest: The authors declare no conflict of interest.

\section{Appendix A}

Table A1. Parametric Value of NN.

\begin{tabular}{cc}
\hline Parameters & Value \\
\hline Controller training Epochs & 50 \\
Controller Training sample & 100 \\
Sampling Interval (sec) & 0.05 \\
Maximum interval value (sec) & 20 \\
Minimum interval value (sec) & 5 \\
Controller training segment & 50 \\
Number of hidden layers & 2 \\
No. of delayed Plant Inputs & 1 \\
No. of delayed Plant outputs & 1 \\
\hline
\end{tabular}

\section{References}

1. Wu, W.; Chen, Y.; Fei, Y.; Zhen, H.; Zhou, B.; Wang, Z.; Chen, W. A novel damping strategy for low-frequency oscillation suppression with MMC-type unified power flow controller. In Proceedings of the IEEE International Conference on Industrial Technology, Lyon, France, 20-22 February 2018.

2. Gandoman, F.H.; Ahmadi, A.; Sharaf, A.M.; Siano, P.; Pou, J.; Hredzak, B.; Agelidis, V.G. Review of FACTS technologies and applications for power quality in smart grids with renewable energy systems. Renew. Sustain. Energy Rev. 2018, 82, 502-514. [CrossRef]

3. Kannayeram, G.; Manoharan, P.S.; Iruthayarajan, M.W.; Sivakumar, T. UPFC damping controller design using multi-objective evolutionary algorithms. Int. J. Bus. Intell. Data Min. 2018, 13, 52-74. [CrossRef]

4. Fortes, E.; Macedo, L.; Araujo, P.B.; Romero, R. A VNS algorithm for the design of supplementary damping controllers for small-signal stability analysis. Int. J. Electr. Power Energy Syst. 2018, 94, 41-56. [CrossRef]

5. Banaei, M.R.; Toloue, H.; Kazemi, F.M.; Oskuee, M.R.J. Damping of power system oscillations using imperialist competition algorithm in power system equipped by HVDC. Int. J. Ain Shams Eng. J. 2015, 6, 75-85. [CrossRef]

6. Martins, L.F.B.; Araujo, P.B.; De Vargas Fortes, E.; Macedo, L.H. Design of the PI-UPFC-POD and PSS Damping Controllers Using an Artificial Bee Colony Algorithm. J. Control Autom. Electr. Syst. 2017, 28, 762-773. [CrossRef]

7. Shahriar, M.S.; Shafiullah, M.; Rana, M.J. Stability enhancement of PSS-UPFC installed power system by support vector regression. Electr. Eng. 2018, 100, 1601-1612. [CrossRef]

8. Shojaeian, S.; Soltani, J.; Arab Markadeh, G. Damping of low-frequency oscillations of multi-machine multi-UPFC power systems, based on adaptive input-output feedback linearization control. IEEE Trans. Power Syst. 2012, 27, 1831-1840. [CrossRef]

9. Esmaili, M.R.; Khodabakhshian, A.; Bornapour, M. A new coordinated design of UPFC controller and PSS for improvement of power system stability using CPCE algorithm. In Proceedings of the IEEE Conference on Electrical Power and Energy EPEC, Ottawa, ON, Canada, 12-14 October 2016.

10. Pandey, R.K.; Gupta, D.K. Knowledge domain states mapping concept for controller tuning in an interconnected power network. Int. J. Electr. Power Energy Syst. 2016, 80, 160-170. [CrossRef]

11. Tavakoli, A.R.; Seifi, A.R.; Arefi, M.M. Fuzzy-PSS and fuzzy neural network non-linear PI controller-based SSSC for damping inter-area oscillations. Trans. Inst. Meas. Control 2016, 40, 733-745. [CrossRef]

12. Moravej, Z.; Pazoki, M.; Khederzadeh, M. New Pattern-Recognition Method for Fault Analysis in Transmission Line With UPFC. IEEE Trans. Power Deliv. 2015, 30, 1231-1242. [CrossRef]

13. Mahmud, M.A.; Pota, H.R.; Hossain, M.J. Full-order nonlinear observer-based excitation controller design for interconnected power systems via exact linearization approach. Int. J. Electr. Power Energy Syst. 2012, 41, 54-62. [CrossRef] 
14. Parimi, A.M.; Elamvazuthi, I.; Kumar, A.V.P.; Cherian, V. Fuzzy logic based control for IPFC for damping low-frequency oscillations in the multimachine power system. In Proceedings of the 2015 IEEE IAS Joint Industrial and Commercial Power Systems/Petroleum and Chemical Industry Conference (ICPSPCIC), Hyderabad, India, 19-21 November 2015.

15. Singh, B.; Mukherjee, V.; Tiwari, P. A survey on impact assessment of DG and FACTS controllers in power systems. Renew. Sustain. Energy Rev. 2015, 42, 846-882. [CrossRef]

16. El-Zonkoly, A. Optimal sizing of SSSC controllers to minimize transmission loss and a novel model of SSSC to study transient response. Electr. Power Syst. Res. 2008, 78, 1856-1864. [CrossRef]

17. Rodríguez, O.; Medina, A.; Andersson, G. Closed-form analytical characterization of non-linear oscillations in power systems incorporating a unified power flow controller. IET Gener. Transm. Distrib. 2015, 9, 1019-1032. [CrossRef]

18. Wang, H. A unified model for analysis of FACTS Devices in Damping Power System Oscillations. Part III: Unified Power Flow Controller. IEEE Trans. Power Deliv. 2000, 15, 978-983. [CrossRef]

19. Torkzadeh, R.; Nasrazadani, H.; Aliabad, A.D. A genetic algorithm optimized fuzzy logic controller for UPFC in order to damp of low-frequency oscillations in power systems. In Proceedings of the 2014 22nd Iranian Conference on Electrical Engineering (ICEE), Tehran, Iran, 20-22 May 2014.

20. Li, Z.; Xia, Y.; Su, C.Y.; Deng, Y.; Fu, J.; He, W. Missile guidance Law Based on robust model predictive control using Neural Network Optimization. IEEE Trans. Neural Netw. Learn. Syst. 2015, 26, 1803-1809. [CrossRef] [PubMed]

21. Xu, R.; Tao, Y.; Lu, Z.; Zhong, Y. Attention-Mechanism-Containing Neural networks for high resolution remote sensing image classification. Electronics 2018, 10, 1602. [CrossRef]

22. Barone, E.R.; Salerno, V.; Siniscalchi, S.M. An introductory study on deep neural networks for high resolution areal images. AIP Conf. Proc. 2013, 1558, 1232. [CrossRef]

23. Sinniscalchi, S.M.; Salerno, V.M. Adaptation to new microphones using artificial neural networks with trainable activation functions. IEEE Trans. Neural Netw. Learn. Syst. 2017, 28, 1959-1965. [CrossRef]

24. Hautamaki, V.; Sinniscalchi, S.M.; Behravan, H.; Salerno, V.M.; Kukanov, I. Boosting universal speech attributes classification with deep Neural Network for foreign accent characterization. In Proceedings of the 16th Annual Conference of the International Speech Communication Association, Dresden, Germany, 6-10 September 2015.

25. Fu, Y.; Chai, T. Neural Network Based nonlinear adaptive dynamical decoupling control. IEEE Trans. Neural Netw. 2007, 18, 921-925. [CrossRef]

26. Zeb, K.; Mehmood, C.A.; Khan, B.; Ali, S.M.; Jadoon, A.M.; Uddin, W. Fault tolerant speed regulation of induction motor using artificial neural network. In Proceedings of the IEEE Conference on Emerging Technologies, Peshawar, Pakistan, 19-20 December 2015.

27. Chae, S.; Kwon, S.; Lee, D. Predicting infectious Disease Using Deep learning and Big Data. Int. J. Environ. Res. Public Health 2018, 15, 1596. [CrossRef] [PubMed]

28. Salerno, V.M.; Rabbeni, G. An Extreme learning machine approach to effective energy disaggregation. Electronics 2018, 7, 235. [CrossRef]

29. Douratsos, I.; Gomm, J.B. Neural Network based model reference adaptive control for process with time delay. Int. J. Inf. Syst. Sci. 2006, 3, 161-179.

(C) 2019 by the authors. Licensee MDPI, Basel, Switzerland. This article is an open access article distributed under the terms and conditions of the Creative Commons Attribution (CC BY) license (http://creativecommons.org/licenses/by/4.0/). 